\title{
Internal dynamics of semiflexible polymers with active noise
}

Thomas Eisenstecken, Gerhard Gompper, and Roland G. Winkler

Citation: The Journal of Chemical Physics 146, 154903 (2017); doi: 10.1063/1.4981012

View online: http://dx.doi.org/10.1063/1.4981012

View Table of Contents: http://aip.scitation.org/toc/jcp/146/15

Published by the American Institute of Physics

\section{Articles you may be interested in}

Perspective: Dissipative particle dynamics

The Journal of Chemical Physics 146, 150901150901 (2017); 10.1063/1.4979514

Spontaneous beating and synchronization of extensile active filament

The Journal of Chemical Physics 146, 154901154901 (2017); 10.1063/1.4979946

Brownian dynamics of confined suspensions of active microrollers

The Journal of Chemical Physics 146, 134104134104 (2017); 10.1063/1.4979494

Electrolyte solutions at curved electrodes. I. Mesoscopic approach

The Journal of Chemical Physics 146, 154703154703 (2017); 10.1063/1.4979947

Pattern, growth, and aging in aggregation kinetics of a Vicsek-like active matter model

The Journal of Chemical Physics 146, 044902044902 (2017); 10.1063/1.4974256

Directed motion from particle size oscillations inside an asymmetric channel

The Journal of Chemical Physics 146, 154103154103 (2017); 10.1063/1.4979984

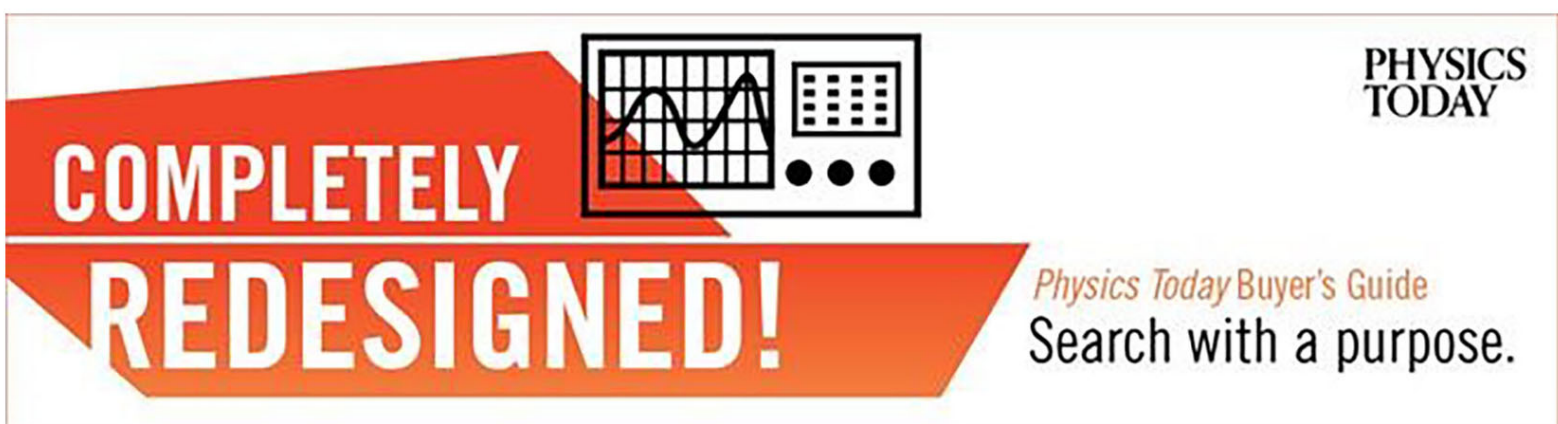




\title{
Internal dynamics of semiflexible polymers with active noise
}

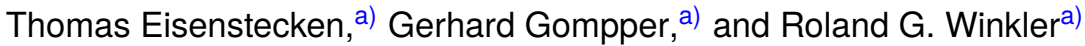 \\ Theoretical Soft Matter and Biophysics, Institute of Complex Systems and Institute for Advanced Simulation, \\ Forschungszentrum Jülich, D-52425 Jülich, Germany
}

(Received 3 March 2017; accepted 3 April 2017; published online 20 April 2017)

\begin{abstract}
The intramolecular dynamics of flexible and semiflexible polymers in response to active noise is studied theoretically. The active noise may either originate from interactions of a passive polymer with a bath of active Brownian particles or the polymer itself is comprised of active Brownian particles. We describe the polymer by the continuous Gaussian semiflexible-polymer model, taking into account the finite polymer extensibility. Our analytical calculations predict a strong dependence of the polymer dynamics on the activity. In particular, active semiflexible polymers exhibit a crossover from a bending elasticity-dominated dynamics at weak activity to that of flexible polymers at strong activity. The endto-end vector correlation function decays exponentially for times longer than the longest polymer relaxation time. Thereby, the polymer relaxation determines the decay of the correlation function for long and flexible polymers. For shorter and stiffer polymers, the relaxation behavior of individual active Brownian particles dominates the decay above a certain activity. The diffusive dynamics of a polymer is substantially enhanced by the activity. Three regimes can be identified in the mean square displacement for sufficiently strong activities: an activity-induced ballistic regime at short times, followed by a Rouse-type polymer-specific regime for any polymer stiffness, and free diffusion at long times, again determined by the activity. Published by AIP Publishing. [http://dx.doi.org/10.1063/1.4981012]
\end{abstract}

\section{INTRODUCTION}

Active matter systems exhibit a wide spectrum of fascinating phenomena, such as the activity-driven phase separation or large-scale collective motion, emerging from the intrinsic nonequilibrium character of their constituents. ${ }^{1-9}$ Thereby, the agents of active matter convert either internal chemical energy into directed motion or utilize energy from the environment. ${ }^{10}$ Prototype examples of active matter are omnipresent in biology and range from the cytoskeleton in living cells ${ }^{2,5,11-18}$ to algae, sperm, and bacteria. ${ }^{2,6,19}$ Moreover, various concepts have been put forward for the design of synthetic active particles. ${ }^{7,20-25}$ Pure active systems show already fascinating nonequilibrium phenomena, but mixtures of active and passive objects ${ }^{26}$ exhibit even more astonishing features, such as collective interface propagation, ${ }^{27}$ and provide a route to switchable self-assembly ${ }^{28}$ and microrheological measurements. ${ }^{29}$ Moreover, an enhanced effective tracer motion has been observed in active suspensions. ${ }^{30,31}$ An eminent example of a mixed active-passive system is an eukaryotic cell with the active cytoskeleton and an embedded large variety of passive colloidal and polymeric objects. Here, the active matrix modifies the structural and dynamical properties of the passive objects to a yet not fully explored extent. Specifically, an enhanced random motion of tracer particles, compared with the thermal Brownian motion, has been observed. ${ }^{32}$ Other experiments indicate an influence of active forces on the dynamics of polymers such as those by

\footnotetext{
a)Electronic addresses: t.eisenstecken@fz-juelich.de; g.gompper@fzjuelich.de; and r.winkler@fz-juelich.de
}

microtubuli ${ }^{33}$ or actin filaments ${ }^{34}$ on the motion of chromosomal loci in simple organisms ${ }^{35,36}$ or the chromatin dynamics in eukaryotes. $^{37}$

In this article, we study the dynamical properties of linear active semiflexible polymers. In particular, we want to shed light onto the influence of activity on their internal dynamics. A major goal is to achieve analytical results; therefore, we adopt the Gaussian semiflexible polymer model ${ }^{38-40}$ exposed to active noise. ${ }^{10,41-44}$ As shown in Ref. 10, the activity can be interpreted in two ways (cf. Fig. 1). On the one hand, our polymer can be considered as comprised of active monomers, e.g., active Brownian particles (ABPs). ${ }^{10,44-47}$ On the other hand, the active force may originate from interactions with uncorrelated surrounding ABPs; hence, the polymer corresponds to a passive polymer dissolved in an active bath. ${ }^{42,43,48}$ Thereby, ABPs are a well established model to study active matter. ${ }^{4,20,49-55}$

The connectivity of active particles, as in linear chains ${ }^{10,44,46-48,56-67}$ or other arrangements, ${ }^{68}$ gives rise to particular interesting conformational and dynamical features. Examples are provided in Refs. 10, 42, 48, and 69, where conformations of passive polymers embedded in a bath of active Brownian particles are analysed. Specifically, flexible polymers swell with increasing activity, whereas semiflexible filaments shrink with increasing activity, i.e., activity leads to a softening of the polymers, and only for large activities the semiflexible polymers swell again, interestingly in the same manner as flexible polymers. ${ }^{10}$ Shrinkage has also be observed in simulation studies of two-dimensional active selfavoiding flexible polymers, ${ }^{45}$ which has been attributed to the activity-induced encaging by neighboring ABPs and may thus be particular for two-dimensional ABPs. Moreover, activity 

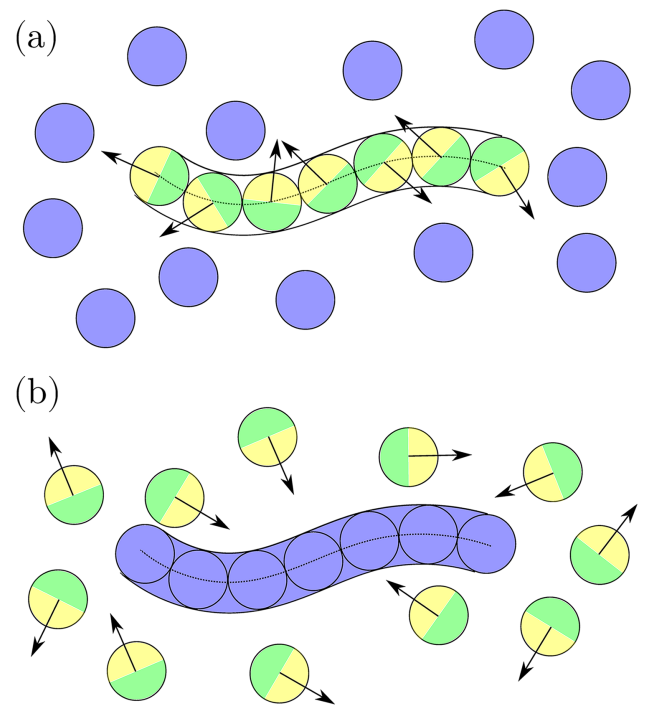

FIG. 1. Illustration of (a) a polymer comprised of $A B P$ monomers in a solution of passive particles and (b) a passive polymer in a solution of ABPs.

significantly affects the polymer kinetics, which is reflected in an accelerated looping dynamics. ${ }^{42,57}$

The dynamics of dumbbells ${ }^{44}$ and linear ${ }^{41,43,58,70}$ chains of ABPs have been studied analytically. In Ref. 58, the active force is transverse to the polymer contour, Refs. 43 and 70 consider Rouse and Zimm type models, and in Ref. 41 a Rouse polymer embedded in a viscoelastic medium is studied applying the formalism of fractional derivatives. In all these cases, a fundamental polymer property, namely, the finite contour length and, hence, the finite polymer extensibility has been neglected.

The considerations of this article reach beyond these previous studies by specifically accounting for the finite polymer extensibility. As we have shown in Ref. 10, finite extensibility drastically changes the polymer conformational properties and the relaxation times, in particular for semiflexible polymers. As discussed above, semiflexible polymers shrink over a wide range of activities compared to the passive case. Moreover, the relaxation times decrease with increasing activity, i.e., dynamical processes are accelerated. Our calculations show that this is a consequence of the constant contour length of a polymer, which implies that this constraint has to be taken into account in analytical considerations. ${ }^{10}$ Here, we will discuss specifically the relaxation behavior of semiflexible active polymers as well as various mean square displacements (MSDs). The relaxation behavior depends significantly on the number of active sites along the polymer contour. For a large number of active sites, the decay of the end-to-end vector correlation function is determined by the relaxation times of the polymer independent of its stiffness. In the case of a small number of active sites, a crossover follows from a decay determined by the polymer relaxation times to a decay governed by the rotational diffusion of an individual ABP. As already noted earlier, ${ }^{41,42,58}$ we find a substantially enhanced diffusion of the active polymers. Three regimes can be identified, each determined by the activity: a ballistic regime for short times, a Rouse-type polymer specific regime for intermediate times, and free diffusion for long times. Thereby, flexible and semiflexible polymers exhibit the same time dependence $(\sqrt{t})$ in the intermediate regime as a consequence of the activity-induced softening of stiff polymers.

The paper is organized as follows. Section II describes the model of the active polymer and presents the equations of motion and their solution. Moreover, the dependence of the mode numbers and relaxation times on the activity is addressed. In Sec. III, results for the polymer end-to-end vector correlation function are presented for various polymer lengths and stiffness. Polymer mean square displacements are discussed in Sec. IV. Section V summarizes the major results of our study. The Appendix presents more detailed analytical calculations and results.

\section{ACTIVE POLYMER MODEL}

\section{A. Equation of motion}

We utilize the Gaussian semiflexible polymer model, ${ }^{38,71,72}$ where the polymer is considered as a continuous, differentiable space curve $\boldsymbol{r}(s, t)$, with the contour coordinate $s$ along the linear chain of length $L(-L / 2<s<L / 2)$ and the time $t$. The equation of motion of $\boldsymbol{r}(s, t)$ is given by the Langevin equation ${ }^{10,40,73-76}$

$$
\begin{aligned}
\frac{\partial}{\partial t} \boldsymbol{r}(s, t)= & \boldsymbol{v}(s, t)+\frac{1}{\gamma} \boldsymbol{\Gamma}(s, t) \\
& +\frac{k_{B} T}{\gamma}\left(2 \lambda \frac{\partial^{2}}{\partial s^{2}} \boldsymbol{r}(s, t)-\epsilon \frac{\partial^{4}}{\partial s^{4}} \boldsymbol{r}(s, t)\right),
\end{aligned}
$$

with the boundary conditions

$$
\begin{gathered}
{\left[2 \lambda \frac{\partial}{\partial s} \boldsymbol{r}(s, t)-\epsilon \frac{\partial^{3}}{\partial s^{3}} \boldsymbol{r}(s, t)\right]_{s= \pm L / 2}=0,} \\
{\left[2 \lambda_{0} \frac{\partial}{\partial s} \boldsymbol{r}(s, t) \pm \epsilon \frac{\partial^{2}}{\partial s^{2}} \boldsymbol{r}(s, t)\right]_{s= \pm L / 2}=0 .}
\end{gathered}
$$

The influence of the activity is captured by assigning the velocity $\boldsymbol{v}(s, t)$ to the point $\boldsymbol{r}(s, t)$. Thereby, $\boldsymbol{v}$ is a non-Markovian but Gaussian stochastic process with zero mean and the correlation function $^{6,8,10,43,44}$

$$
\left\langle\boldsymbol{v}(s, t) \cdot \boldsymbol{v}\left(s^{\prime}, t^{\prime}\right)\right\rangle=v_{0}^{2} l e^{-\gamma_{R}\left|t-t^{\prime}\right|} \delta\left(s-s^{\prime}\right) .
$$

Here, $v_{0}$ is the propulsion velocity and $\gamma_{R}$ the damping factor for the rotational motion of an ABP. The latter is related to the rotational diffusion coefficient $D_{R}$ of a passive particle via $\gamma_{R}=(d-1) D_{R}$, where $d$ denotes the dimension. In this article, we restrict ourselves to three dimensions. More details on the derivation of Eq. (4) are presented in Ref. 10. Note that we introduce the length scale $l$ in Eq. (4) in the continuum representation of the semiflexible polymer. As discussed in Ref. 10, in a touching-bead model of a discrete polymer, $l$ represents the bead diameter and bond length. Hence, the ratio $L / l$ can be interpreted as the number of uniformly distributed active sites along the polymer. In the following, if not indicated differently, we consider a polymer with $L / l=10^{3}$. The colored noise $\boldsymbol{v}(s, t)$ may originate from two sources. On the one hand, the polymer may be comprised of active Brownian particles, which change their orientation and propagation direction in a diffusive manner independent of their translational motion. ${ }^{6-8,10,50,51,53} \mathrm{On}$ 
the other hand, the passive polymer may be embedded in a solution of such active Brownian particles and the interaction between them and the polymer is described by the colored noise of Eq. (4) (cf. Fig. (1)). 6,10,42-44,69,77

The stochastic process $\boldsymbol{\Gamma}(s, t)$ for the translational motion of $\boldsymbol{r}(s, t)$ is assumed to be stationary, Markovian, and Gaussian with zero mean and the second moments,

$$
\left\langle\Gamma_{\alpha}(s, t) \Gamma_{\beta}\left(s^{\prime}, t^{\prime}\right)\right\rangle=2 \gamma k_{B} T \delta_{\alpha \beta} \delta\left(s-s^{\prime}\right) \delta\left(t-t^{\prime}\right),
$$

where $T$ is the temperature, $k_{B}$ the Boltzmann constant, $\gamma$ the translational friction coefficient per length, and $\alpha, \beta \in\{x, y, z\}$. The translational friction coefficient is related to the translational diffusion coefficient $D_{T}$ of an individual $\mathrm{ABP}$ via $D_{T}=k_{B} T / \gamma l$.

The terms in the brackets of Eq. (1) represent the entropic degrees of freedom, with the stretching coefficient $\lambda$, and restrictions by an intrinsic bending stiffness, with the bending coefficient $\epsilon$. The latter, together with the coefficient $\lambda_{0}$, are determined via constraints for bond lengths and bending restrictions. ${ }^{39,78}$ Adopting the results for calculations of continuous semiflexible polymers exposed to external forces, ${ }^{39}$ we set $\epsilon=3 / 4 p$ and $\lambda_{0}=3 / 4$ for a polymer in three dimensions, where $p=1 / 2 l_{p}$ and $l_{p}$ is the persistence length. Within a mean-field approach for the bond constraints, we impose the global condition ${ }^{10}$

$$
\int_{-L / 2}^{L / 2}\left\langle\left(\frac{\partial \boldsymbol{r}(s, t)}{\partial s}\right)^{2}\right\rangle d s=L
$$

to capture the inextensibility of a polymer and to determine the stretching coefficient (Lagrangian multiplier) $\lambda$.

\section{B. Solution of equation of motion: Eigenfunction expansion}

The solution of the linear equation of motion (1) follows by an eigenfunction expansion ${ }^{10,73,79}$

$$
\boldsymbol{r}(s, t)=\sum_{n=0}^{\infty} \chi_{n}(t) \varphi_{n}(s),
$$

with the eigenfunctions $\varphi_{n}(s)$ of the equation

$$
\epsilon k_{B} T \frac{d^{4}}{d s^{4}} \varphi_{n}(s)-2 \lambda k_{B} T \frac{d^{2}}{d s^{2}} \varphi_{n}(s)=\xi_{n} \varphi_{n}(s)
$$

and the mode amplitudes $\chi_{n}(t)$. Explicitly, the eigenfunctions $\operatorname{read}^{73,79}$

$$
\begin{aligned}
\varphi_{0} & =\sqrt{\frac{1}{L}} \\
\varphi_{n}(s) & =\sqrt{\frac{c_{n}}{L}}\left(\zeta_{n}^{\prime} \frac{\sinh \zeta_{n}^{\prime} s}{\cosh \zeta_{n}^{\prime} L / 2}+\zeta_{n} \frac{\sin \zeta_{n} s}{\cos \zeta_{n} L / 2}\right), n \text { odd }, \\
\varphi_{n}(s) & =\sqrt{\frac{c_{n}}{L}}\left(\zeta_{n}^{\prime} \frac{\cosh \zeta_{n}^{\prime} s}{\sinh \zeta_{n}^{\prime} L / 2}-\zeta_{n} \frac{\cos \zeta_{n} s}{\sin \zeta_{n} L / 2}\right), n \text { even, }
\end{aligned}
$$

with

$$
\zeta_{n}^{\prime 2}-\zeta_{n}^{2}=\frac{2 \lambda}{\epsilon}, \xi_{0}=0, \xi_{n}=k_{B} T\left(\epsilon \zeta_{n}^{4}+2 \lambda \zeta_{n}^{2}\right) .
$$

The constants $c_{n}$ are normalization coefficients and the wave numbers $\zeta_{n}$ and $\zeta_{n}^{\prime}$ are specified by the boundary conditions Eqs. (2) and (3). $\varphi_{0}$ accounts for the polymer's translational motion. $\xi_{n}$ are the eigenvalues, which depend on the mode number and, in particular, the stretching coefficient $\lambda$, which itself is a function of activity. ${ }^{10,44}$

Due to the odd and even parity eigenfunctions (10) and (11), the boundary conditions (2) and (3) lead to independent even and odd transcendental equations for the mode numbers. ${ }^{73}$ As for passive polymers, the relation

$$
(2 n-1) \pi / 2 \leq \zeta_{n} L \leq n \pi, \quad n>1
$$

holds, with $\zeta_{n}$ values between the wave numbers of a semiflexible $\left(\zeta_{n} L=(2 n-1) \pi / 2, p L \rightarrow 0\right)$ and a flexible $\left(\zeta_{n} L\right.$ $=n \pi, p L \rightarrow \infty)$ polymer. Note that $\zeta_{0}=0$ is a solution corresponding to the translation of the whole polymer. Since the boundary conditions depend on $\lambda$, the wave numbers are functions of activity and are therefore different from those of passive polymers. Numerical results for the first few wave numbers are presented in Fig. 2 (cf. discussion in Sec. II C).

The stationary-state solution of the mode amplitudes is

$$
\begin{aligned}
& \chi_{0}(t)=\chi_{0}(0)+\int_{0}^{t}\left(\boldsymbol{v}_{0}\left(t^{\prime}\right)+\frac{1}{\gamma} \boldsymbol{\Gamma}_{0}\left(t^{\prime}\right)\right) d t^{\prime} \\
& \chi_{n}(t)=\int_{-\infty}^{t} e^{-\left(t-t^{\prime}\right) / \tau_{n}}\left(\boldsymbol{v}_{n}\left(t^{\prime}\right)+\frac{1}{\gamma} \boldsymbol{\Gamma}_{n}\left(t^{\prime}\right)\right) d t^{\prime}
\end{aligned}
$$

$\boldsymbol{v}_{n}(t)$ and $\boldsymbol{\Gamma}_{n}(t)$ are the normal mode amplitudes of the velocity $\boldsymbol{v}(s, t)$ and stochastic force $\boldsymbol{\Gamma}(s, t)$, respectively, in terms of the eigenfunctions $\varphi_{n}(s)$, analogously to Eq. (7), and $\tau_{n}$ are the relaxation times ${ }^{10}(n>0)$,

$$
\tau_{n}=\frac{\gamma}{\xi_{n}}=\frac{\gamma}{k_{B} T\left(\epsilon \zeta_{n}^{4}+2 \lambda \zeta_{n}^{2}\right)} .
$$

Here, a remark on the choice of the initial time is in order. Since the stochastic process (4) is non-Markovian, the solution of the mode amplitudes (Eqs. (14) and (15)) depends on the initial condition. We focus on the dynamics in the stationary state only, with the polymer exposed to the random forces from a starting time $t_{0} \rightarrow-\infty$ on.

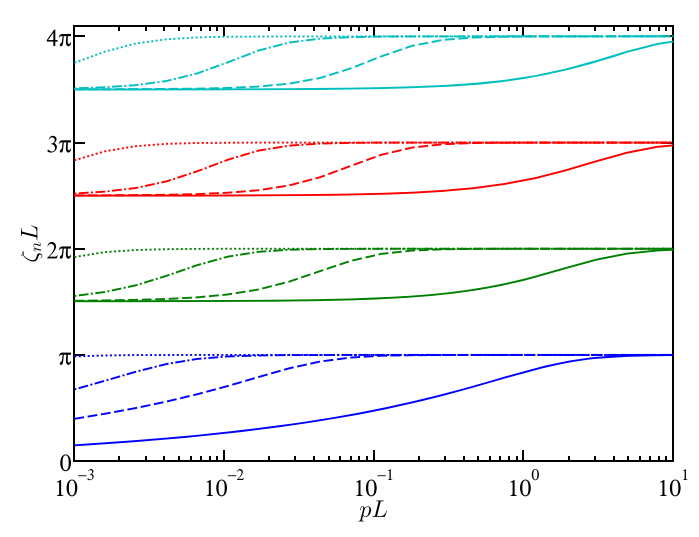

FIG. 2. The first four wave numbers $\zeta_{n}$ ( $n>0$, bottom to top) as a function of the persistence length $(p L)$ for the Péclet numbers $P e=0$ (solid), $P e$ $=3$ (dashed), $P e=10$ (dashed-dotted), and $P e=30$ (dotted). In the limit $p L \rightarrow \infty$ (flexible polymer), the asymptotic values $\zeta_{n} L=n \pi$ are assumed, whereas for $p L \rightarrow 0$ (semiflexible polymer), the values $\zeta_{n} L=(2 n-1) \pi / 2$ are approached $(n>1)$. $\zeta_{1}$ converges to zero in the rodlimit $p L \rightarrow 0$. The ratio $L / l$ $=10^{3}$. 


\section{Stretching coefficient $\lambda$}

Inserting the eigenfunction expansion (7) in Eq. (6) yields the equation for the stretching coefficient $\lambda$,

$$
\sum_{n=1}^{\infty}\left(\frac{3 k_{B} T}{\gamma} \tau_{n}+\frac{v_{0}^{2} l}{1+\gamma_{R} \tau_{n}} \tau_{n}^{2}\right) \int_{-L / 2}^{L / 2}\left(\frac{d \varphi_{n}(s)}{d s}\right)^{2} d s=L .
$$

An approximate equation for a flexible polymer is provided in Ref. 10. A more precise approximation is presented in the Appendix. The relaxation times (16) depend on $\lambda$, both explicitly and implicitly via the wave numbers. The simultaneous solution of Eq. (17) and the equations for the wave numbers yields $\zeta_{n}$ and $\lambda$. Numerical solutions of these equations for the wave numbers and the stretching coefficient are provided in Figs. 2 and 3, respectively, for various persistence lengths, i.e., $p L=L / 2 l_{p}$ values, and Péclet numbers $P e$. Here, the Péclet number

$$
P e=\frac{v_{0}}{D_{R} l}
$$

is introduced to characterize the activity, where $D_{R}$ is the rotational diffusion coefficient of an ABP. Moreover, we introduce the ratio

$$
\Delta=\frac{D_{T}}{D_{R} l^{2}}
$$

between the translational and rotational diffusion coefficients. In the case of a thermal system, the two diffusion coefficients are related, with $\Delta=1 / 3$ for a spherical colloid of diameter $l$ in solution. Microswimmers may exhibit a faster than thermal diffusive rotational dynamics due to, e.g., tumbling motion. ${ }^{6}$ If not otherwise indicated, we will consider the ratio $\Delta=0.3$ in the following.

The wave numbers $\zeta_{n}$ exhibit a pronounced dependence on $P e$, specifically, the crossover from semiflexible to flexible polymer behavior is affected, with a shift of the inflection point to smaller $p L$ values with increasing $P e$ (cf. Fig. 2). Hence, the flexible modes dominate the polymer dynamics even for small $p L$ values at large Péclet numbers.

As already discussed in Ref. 10, the scaled stretching coefficient $\mu=2 \lambda / 3 p$ depends strongly on the Péclet number and increases with increasing $P e$ as shown in Fig. 3. Thereby, the actual dependence on $P e$ varies with the persistence length.

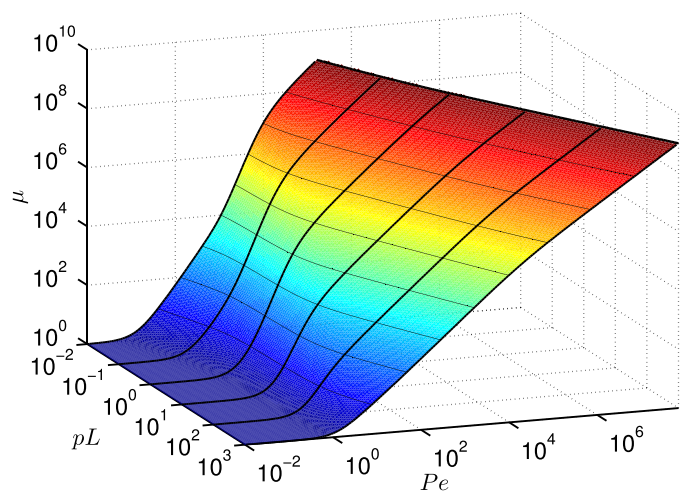

FIG. 3. Scaled stretching coefficient $\mu=2 \lambda / 3 p$ as a function of the Péclet number and the persistence length $l_{p}\left(p L=L / 2 l_{p}\right)$. The colors indicate the value of $\mu$.
The increase of $\lambda$ with increasing $P e$ reflects the tendency of the active force to stretch the polymer, which is compensated by an increase of the mean bond force in order to maintain the constant average contour length in our approach.

\section{Relaxation times}

The polymer relaxation times $\tau_{n}$ follow via Eq. (16) from the wave numbers $\zeta_{n}$. Figure 4 illustrates the dependence of $\tau_{n}$ on the persistence length and Péclet number for various modes. Analogous to the wave numbers, the $\tau_{n}(n>1)$ exhibit three distinctive regimes. In the limit of flexible polymers $(p L \rightarrow \infty)$, the relaxation times are proportional to $L^{2} / n^{2}$ corresponding to those of the Rouse model, ${ }^{80}$ more precisely,

$$
\tau_{n}=\frac{\tau_{R}}{\mu n^{2}}
$$

with the Rouse relaxation time $\tau_{R}=\gamma L^{2} / 3 \pi k_{B} T p .{ }^{10}$ For stiff polymers $(p L \rightarrow 0)$, the bending modes dominate, resulting in relaxation times proportional to $L^{4} /(2 n-1)^{4}(n>1){ }^{76,81}$ In the intermediate regime, both bending and stretching contribute to the relaxation times, with a pronounced influence of activity. The latter leads to a shift of the maxima of the curves to smaller $p L$ values. This again reflects the decreasing importance of polymer stiffness on the relaxation behavior with increasing $P e$. Interestingly, the relaxation times develop a plateau-like regime over several orders of $p L$ values. Here, the respective $\tau_{n}$ are essentially independent of the persistence length. Moreover, the plateau values of $\tau_{1}$ for $p L \rightarrow 0$, which correspond to the rotational diffusive motion of a rod-like polymer, decrease with increasing $P e$. A more extensive discussion of the dependence of the longest relaxation time on the Péclet number is presented in Ref. 10. The most important message here is that the relaxation times significantly depend on the activity of the polymer due to the finite extensibility of a polymer, an aspect often neglected in theoretical studies.

\section{E. Correlation functions}

The characterisation of the polymer dynamics typically requires correlation functions of the normal-mode amplitudes $\chi_{n}(t)$. Using Eqs. (4) and (5), we can calculate the necessary

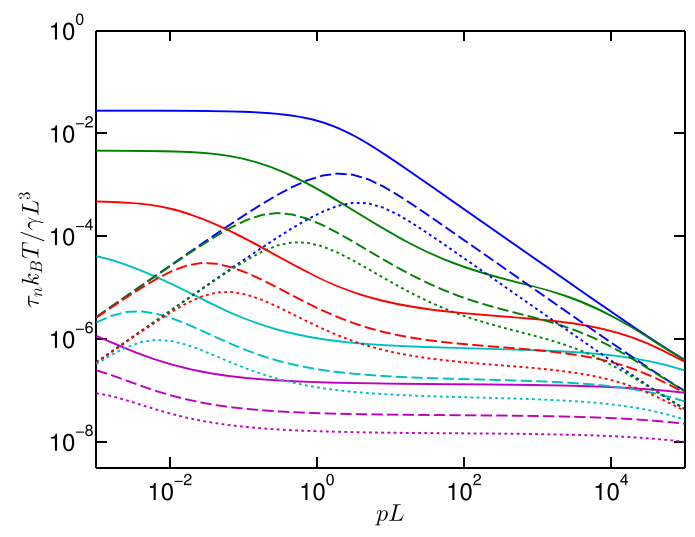

FIG. 4. The first three relaxation times $\tau_{n}$ ( $n=1$ (solid), $n=2$ (dashed), $n=3$ (dotted)) as a function of $p L$ for the Péclet numbers $P e=0$ (blue), $P e=1$ (green), $P e=3$ (red), $P e=10$ (cyan), and $P e=30$ (purple). For the flexible polymer $(p L \rightarrow \infty) \tau_{n} \sim L^{2} / n^{2}(n \geq 1)$, whereas for a semiflexible polymer $(p L \rightarrow 0) \tau_{n} \sim L^{4} /(2 n-1)^{4}(n>1)$. The plateau value of $\tau_{1}$ for $p L \rightarrow 0$ decreases with increasing Péclet number. ${ }^{10}$ 
correlation functions for the amplitudes $\boldsymbol{\Gamma}_{n}(t)$ and $\boldsymbol{v}_{n}(t)$ of the eigenfunction expansion of the random variables $v(s, t)$ and $\boldsymbol{\Gamma}(s, t)$ in terms of $\varphi_{n}$. This yields zero odd moments and the second moments,

$$
\begin{aligned}
\left\langle\boldsymbol{v}_{n}(t) \cdot \boldsymbol{v}_{m}\left(t^{\prime}\right)\right\rangle & =v_{0}^{2} l e^{-\gamma_{R}\left|t-t^{\prime}\right|} \delta_{n m}, \\
\left\langle\boldsymbol{\Gamma}_{n}(t) \cdot \boldsymbol{\Gamma}_{m}\left(t^{\prime}\right)\right\rangle & =6 \gamma k_{B} T \delta\left(t-t^{\prime}\right) \delta_{n m} .
\end{aligned}
$$

The correlation functions of the normal-mode amplitudes are given by

$$
\begin{aligned}
\left\langle\chi_{n}(t) \cdot \chi_{m}\left(t^{\prime}\right)\right\rangle= & \delta_{n m} e^{-\left(t+t^{\prime}\right) / \tau_{n}}\left(\int_{-\infty}^{t} \int_{-\infty}^{t^{\prime}} e^{\left(t_{1}+t_{2}\right) / \tau_{n}}\right. \\
& \times\left\langle\boldsymbol{v}_{n}\left(t_{1}\right) \cdot \boldsymbol{v}_{n}\left(t_{2}\right)\right\rangle d t_{1} d t_{2} \\
& \left.+\int_{-\infty}^{t} \int_{-\infty}^{t^{\prime}} \frac{1}{\gamma^{2}}\left\langle\boldsymbol{\Gamma}_{n}\left(t_{1}\right) \cdot \boldsymbol{\Gamma}_{n}\left(t_{2}\right)\right\rangle d t_{1} d t_{2}\right) \\
= & \delta_{n m}\left(\frac{3 k_{B} T \tau_{n}}{\gamma} e^{-\left|t-t^{\prime}\right| / \tau_{n}}+\frac{v_{0}^{2} l \tau_{n}^{2}}{1-\left(\gamma_{R} \tau_{n}\right)^{2}}\right. \\
& \left.\times\left[e^{-\gamma_{R}\left|t-t^{\prime}\right|}-\gamma_{R} \tau_{n} e^{-\left|t-t^{\prime}\right| / \tau_{n}}\right]\right) .
\end{aligned}
$$

The last equality follows by the insertion of Eqs. (21) and (22) and evaluation of the integrals. ${ }^{10,43,44}$

\section{POLYMER END-TO-END VECTOR CORRELATION FUNCTION}

The correlation function $\left\langle\boldsymbol{r}_{e}(t) \cdot \boldsymbol{r}_{e}(0)\right\rangle$ of the polymer endto-end vector $\boldsymbol{r}_{e}=\boldsymbol{r}(L / 2)-\boldsymbol{r}(-L / 2)$ is given by

$\left\langle\boldsymbol{r}_{e}(t) \cdot \boldsymbol{r}_{e}(0)\right\rangle=4 \sum_{n=1}^{\infty}\left\langle\chi_{2 n-1}(t) \cdot \chi_{2 n-1}(0)\right\rangle \varphi_{2 n-1}^{2}(L / 2)$

in terms of the normal-mode amplitudes (Eq. (23)), where only odd terms contribute. Inserting Eq. (23), we find explicitly

$$
\begin{aligned}
\left\langle\boldsymbol{r}_{e}(t) \cdot \boldsymbol{r}_{e}(0)\right\rangle= & \sum_{n=1}^{\infty} \varphi_{2 n-1}^{2}(L / 2)\left[\frac{4 L^{3}}{\hat{\xi}_{2 n-1}} e^{-t / \tau_{2 n-1}}\right. \\
& +\frac{P e^{2} l^{3}}{9 \Delta^{2} \hat{\xi}_{2 n-1}^{2} l^{6} /\left(4 L^{6}\right)-1} \\
& \left.\times\left(e^{-\gamma_{R} t}-\frac{2 L^{3}}{3 \Delta l^{3} \hat{\xi}_{2 n-1}} e^{-t / \tau_{2 n-1}}\right)\right],
\end{aligned}
$$

where $\hat{\xi}_{n}=p L \mu\left(\zeta_{n} L\right)^{2}+\left(\zeta_{n} L\right)^{4} /(4 p L)$. The decay of the correlation function is governed by two processes, the Brownian motion of the active force $\left(\gamma_{R} t\right)$ and the polymer dynamics $\left(t / \tau_{n}\right)$.

In the limits $\gamma_{R} \tau_{1} \gg 1$ and $t / \tau_{1}>1$, the correlation function exhibits the asymptotic behavior

$$
\left\langle\boldsymbol{r}_{e}(t) \cdot \boldsymbol{r}_{e}(0)\right\rangle=\frac{L^{3}}{\hat{\xi}_{1}} e^{-t / \tau_{1}}\left(4+\frac{2 P e^{2}}{3 \Delta}\right) \varphi_{1}^{2}(L / 2) \sim\left\langle\boldsymbol{r}_{e}^{2}\right\rangle e^{-t / \tau_{1}} .
$$

For flexible polymers in general, and in particular in the case of stiff polymers for sufficiently large Péclet numbers, where the
Rouse modes dominate over the bending modes, the condition $\gamma_{R} \tau_{1} \gg 1$ is equivalent to

$$
P e \ll\left(\frac{L}{l}\right)^{3 / 2}
$$

when we use the expressions of Eq. (32) or (33) of Ref. 10. Hence, for a large number $L / l$ of active sites along a polymer or small Péclet numbers, the correlation function is determined by the relaxation times of the polymer. Corresponding correlation functions are displayed in Fig. 5 for flexible and semiflexible polymers. Evidently, the decay is exponential for $t / \tau_{1}>1$ and $\tau_{1}$ is the dominant relaxation time. For flexible polymers, the correlation function increases monotonically with increasing Péclet number for all times $t / \tau_{1}$, which reflects the monotonic increase of the mean square end-to-end distance $\left\langle\boldsymbol{r}_{e}^{2}\right\rangle$ with increasing $P e{ }^{10}$ This no longer applies to semiflexible polymers, where a nonmonotonic dependence on $P e$ is observed for the correlation function (cf. Fig. 5(b) for $P e=10^{3}$ ). Again, this is in agreement with the nonmonotonic behavior of $\left\langle\boldsymbol{r}_{e}^{2}\right\rangle$ as a function of the Péclet number. At short times $\left(t / \tau_{1} \ll 1\right)$, various modes and the rotational diffusion $\left(\gamma_{R}\right)$ contribute to the decay of the correlation function, as illustrated in Fig. 5.

In the limit $\gamma_{R} \tau_{1} \ll 1$, the decay of the correlation function is determined by the rotational diffusion of an individual ABP
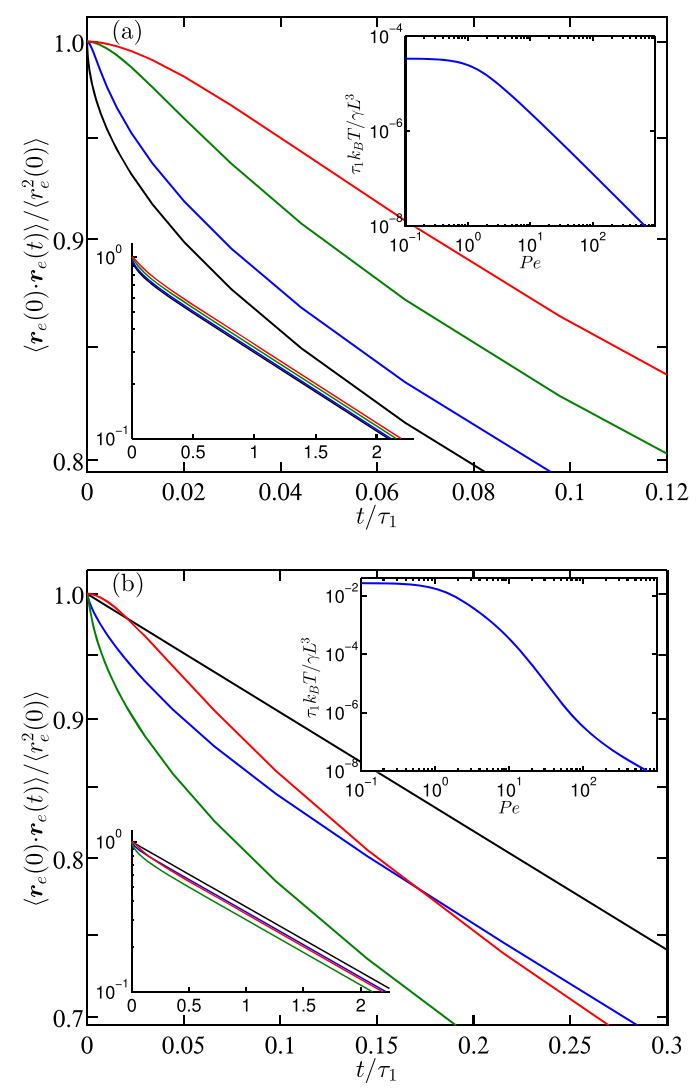

FIG. 5. End-to-end vector correlation functions of (a) flexible polymers with $p L=10^{3}$ and the Péclet numbers $P e=0$ (black), $10^{2}$ (blue), $5 \times 10^{2}$ (green), and $10^{3}$ (red), and (b) semiflexible polymers with $p L=10^{-2}$ and $P e=0$ (black), $2 \times 10^{1}$ (blue), $10^{2}$ (green), and $10^{3}$ (red). The time is scaled by the longest polymer relaxation time. The insets in the upper right corners show the dependence of the longest relaxation time on the Péclet number. The insets in the lower left corners highlight the exponential decay of the correlation function. 


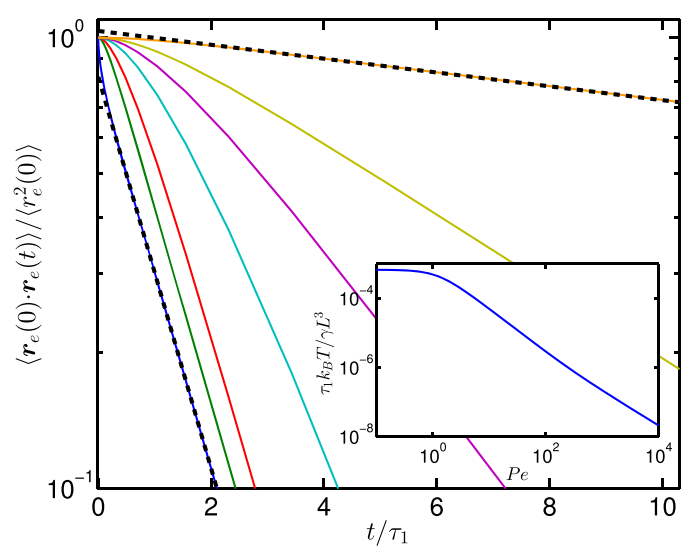

FIG. 6. End-to-end vector correlation functions of flexible polymers with $p L$ $=L / l=50$ for the Péclet numbers $P e=0$ (blue), $5 \times 10^{1}$ (green), $10^{2}$ (red), $2.5 \times 10^{2}$ (cyan), $5 \times 10^{2}$ (purple), $10^{3}$ (yellow), and $5 \times 10^{3}$ (orange). The time is scaled by the longest polymer relaxation time as displayed in the inset. The dashed lines indicate the limits of Eqs. (26) and (29) for low and high Péclet numbers, respectively.

and it is given by (cf. Eq. (25))

$$
\left\langle\boldsymbol{r}_{e}(t) \cdot \boldsymbol{r}_{e}(0)\right\rangle=\frac{4 P e^{2} L^{3}}{9 \Delta^{2}}\left(\frac{L}{l}\right)^{3} e^{-\gamma_{R} t} \sum_{n=1}^{\infty} \frac{\varphi_{2 n-1}^{2}(L / 2)}{\hat{\xi}_{2 n-1}},
$$

which reduces to

$$
\left\langle\boldsymbol{r}_{e}(t) \cdot \boldsymbol{r}_{e}(0)\right\rangle=\frac{P e^{2} L^{2}}{108(\Delta p L \mu)^{2}}\left(\frac{L}{l}\right)^{3} e^{-\gamma_{R} t}
$$

when flexible modes dominate.

Figure 6 shows correlation functions of a (short) flexible polymer with $L / l=50$ active elements and various Péclet numbers. For this choice, condition (27) is violated at larger $\mathrm{Pe}$. Correspondingly, we observe a gradual crossover from the dependence $\left\langle\boldsymbol{r}_{e}(t) \cdot \boldsymbol{r}_{e}(0)\right\rangle \sim e^{-t / \tau_{1}}$ at small Péclet numbers to the decay $\left\langle\boldsymbol{r}_{e}(t) \cdot \boldsymbol{r}_{e}(0)\right\rangle \sim e^{-\gamma_{R} t}$ for large Péclet numbers. The absence or presence of the crossover strongly depends on $L / l$ (cf. Eq. (27)). Hence, for long polymers, where $p L=L / l \gg 1$, we observe only the decay $\left\langle\boldsymbol{r}_{e}(t) \cdot \boldsymbol{r}_{e}(0)\right\rangle \sim e^{-t / \tau_{1}}$ as long as $P e \lesssim 10^{3}$. In the limiting case of an active dumbbell, where $L / l=2$, the decay of the correlation function is already for rather small $\mathrm{Pe}$ governed by the rotational diffusion of the monomers. ${ }^{4}$

As discussed in Sec. II D and Ref. 10, the longest polymer relaxation time $\tau_{1}$ depends strongly on activity. Hence, a part of the activity dependence of the correlation function is absorbed in the scaled time $t / \tau_{1}$ in Figs. 5 and 6. In contrast, Fig. 7 presents correlation functions as a function of $\gamma_{R} t$ for various Péclet numbers. It is obvious that the correlation function decays faster for larger activities. Thereby, the correlation function of semiflexible polymers decays slower than that of flexible polymers for small-to-moderate Péclet numbers, but, in the limit of large Péclet numbers, the decay rates become equal. This once again reflects the transition of a semiflexible to a flexible polymer behavior with increasing activity. ${ }^{10}$

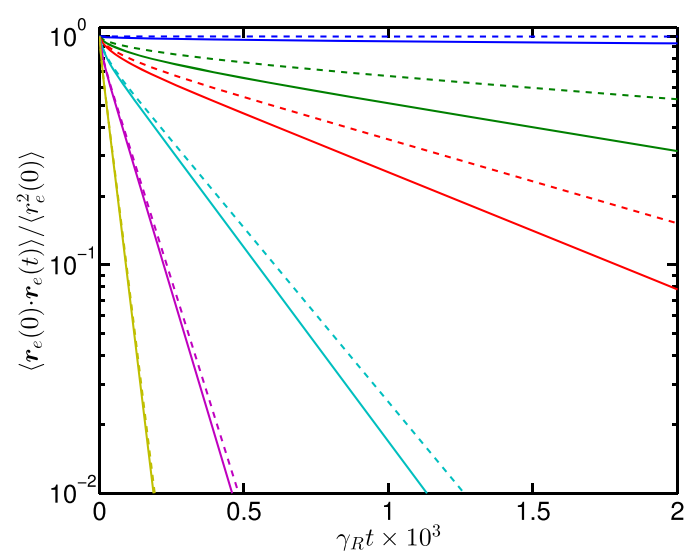

FIG. 7. End-to-end vector correlation functions of a flexible $\left(p L=10^{3}\right.$, solid) and semiflexible $\left(p L=10^{-1}\right.$, dashed) polymers for the Péclet numbers $P e=0$ (blue), $5 \times 10^{1}$ (green), $10^{2}$ (red), $2.5 \times 10^{2}$ (cyan), $5 \times 10^{2}$ (purple), and $10^{3}$ (yellow). The time is scaled by the coefficient $\gamma_{R}=2 D_{R}$ of the rotational Brownian motion.

\section{POLYMER MEAN SQUARE DISPLACEMENT}

The mean square displacement (MSD) of the point $\boldsymbol{r}(s, t)$ is given by

$$
\begin{aligned}
\left\langle\Delta \boldsymbol{r}^{2}(s, t)\right\rangle= & \left\langle(\boldsymbol{r}(s, t)-\boldsymbol{r}(s, 0))^{2}\right\rangle=\varphi_{0}^{2}\left\langle\left(\chi_{0}(t)-\chi_{0}(0)\right)^{2}\right\rangle \\
& +\sum_{n=1}^{\infty} \varphi_{n}^{2}(s)\left\langle\left(\chi_{n}(t)-\chi_{n}(0)\right)^{2}\right\rangle
\end{aligned}
$$

in terms of the mode amplitudes. The first term of the righthand side is the mean square displacement of the center-ofmass $\left\langle\Delta r_{c m}^{2}(t)\right\rangle$ of the polymer and the second term is the displacement of the point $\boldsymbol{r}(s, t)$ with respect to the center of mass. Insertion of the correlation functions (23) yields

$$
\begin{aligned}
\left\langle\Delta \boldsymbol{r}^{2}(s, t)\right\rangle= & \left\langle\Delta \boldsymbol{r}_{c m}^{2}(t)\right\rangle+\sum_{n=1}^{\infty} \varphi_{n}^{2}(s)\left[\frac{6 k_{B} T \tau_{n}}{\gamma}\left(1-e^{-t / \tau_{n}}\right)\right. \\
& \left.+\frac{2 v_{0}^{2} l \tau_{n}^{2}}{1+\gamma_{R} \tau_{n}}\left(1-\frac{e^{-\gamma_{R} t}-\gamma_{R} \tau_{n} e^{-t / \tau_{n}}}{1-\gamma_{R} \tau_{n}}\right)\right]
\end{aligned}
$$

with the center-of-mass mean square displacement ${ }^{10}$

$$
\left\langle\Delta r_{c m}^{2}(t)\right\rangle=\frac{6 k_{B} T}{\gamma L} t+\frac{2 v_{0}^{2} l}{\gamma_{R}^{2} L}\left(\gamma_{R} t-1+e^{-\gamma_{R} t}\right) .
$$

This expression is similar to that of a single active Brownian particle. , $8,20,44$ As a generalization, the total polymer friction coefficient $\gamma L$ appears in the Brownian motion of the center of mass, and the active term contains $L / l$, the number of active sites along the polymer contour. ${ }^{10}$ The latter can be understood as follows. The center-of-mass motion of a polymer is independent of internal forces. Hence, on the level of the center-of-mass, we are left with $L / l$ independent ABPs. In the limit $t \rightarrow \infty$, their MSD is proportional to $v_{0}^{2} /(L / l)$, with the fluctuation $\sqrt{l / L}$ in the number of active sites due to the Gaussian nature of the process.

MSDs of flexible and semiflexible polymers are displayed in Fig. 8 for various Péclet numbers. To obtain a site-independent displacement, $\left\langle\Delta \boldsymbol{r}^{2}(s, t)\right\rangle$ is averaged over $s$, i.e., $\left\langle\overline{\Delta \boldsymbol{r}^{2}}(t)\right\rangle=\int\left\langle\Delta \boldsymbol{r}^{2}(s, t)\right\rangle d s / L$ is considered. For a passive 

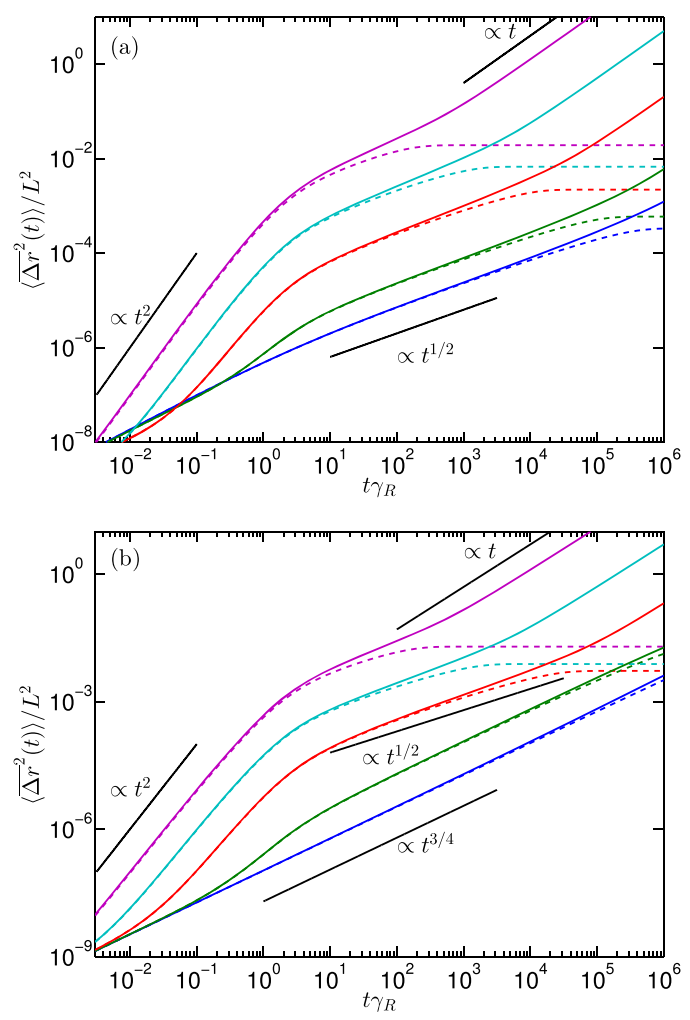

FIG. 8. Mean square displacements of (a) a flexible polymer with $p L=10^{3}$ and (b) a semiflexible polymer with $p L=1$. The Péclet numbers are $P e=0$ (blue), $3 \times 10^{0}$ (green), $2 \times 10^{1}$ (red), $10^{2}$ (cyan), and $5 \times 10^{2}$ (purple). The time is scaled by the factor $\gamma_{R}=2 D_{R}$ of the rotational diffusive movement. The dashed lines correspond to the MSDs in the polymer center-of-mass reference frame.

flexible polymer (Fig. 8(a)), we recover the well-known Rousedependence $\left\langle\overline{\Delta \boldsymbol{r}^{2}}(t)\right\rangle \sim \sqrt{t}$ for $t / \tau_{1} \ll 1$ and a crossover to free diffusion for $t / \tau_{1} \gg 1 .{ }^{80}$ Activity implies a significantly enhanced diffusive motion as already reported before. ${ }^{41,42,58}$ At short times, a specific ballistic regime appears, which is already well pronounced for $P e \approx 20$. For times $\gamma_{R} t \approx 1$, the MSD crosses over to a Rouse-type regime $(\sqrt{t})$ determined by the internal polymer dynamics. Finally, the center-of-mass motion dominates the MSD with a linear increase in time. Thereby, the crossover time $\gamma_{R} t$ from the ballistic to the Rousetype regime shifts to smaller values with increasing Péclet number, a consequence of the decreasing relaxation times $\tau_{n}$ with increasing $P e$ (cf. Fig. 4 and discussion in Ref. 10). Simultaneously, the polymer characteristic regime $\sim \sqrt{t}$ becomes shorter and vanishes for large Péclet numbers. This shortening is a consequence of the finite contour length and is completely missing in a bare Rouse-model-type approach. ${ }^{41-43,58,70}$ The finite contour length leads to an activity-depending relaxation time and, hence, a qualitative different dynamical behavior.

The MSD of passive semiflexible polymers (Fig. 8(b)) exhibits the well-known $\left\langle\overline{\Delta \boldsymbol{r}^{2}}(t)\right\rangle \sim t^{3 / 4}$ dependence for $t / \tau_{1}$ $\ll 1 .^{71,76,82-85}$ As for flexible polymers, activity substantially enhances the diffusive motion. We observe the same sequence of time regimes as for flexible polymers: ballistic motion for $\gamma_{R} t \lesssim 1$ followed by a regime determined by the internal dynamics of the polymer and, finally, the overall diffusion of the polymer for $t / \tau_{1} \gg 1$. However, there is a remarkable feature in the intermediate regime $1<\gamma_{R} t<\gamma_{R} \tau_{1}$. Here, at sufficiently large Péclet numbers, the MSD of semiflexible polymers exhibits the $\sqrt{t}$-dependence characteristic for flexible polymers. This is a consequence of the finite contour length of the polymer captured in the $P e$ dependence of the stretching coefficient $\lambda$. The crossover of the relaxation times from bending-mode dominated to Rouse-mode dominated of flexible polymers gives rise to the appearing change in the sub-diffusive behavior. Such a change does not appear for the stretching coefficient $\mu=1$. In this case, the $t^{3 / 4}$ dependence persists for all $P e$ in the intermediate time regime.

The different regimes of the internal polymer dynamics follow by suitable approximations of Eq. (31), specifically its contribution by the activity

$$
\left\langle\overline{\Delta \boldsymbol{r}_{a}^{2}}(t)\right\rangle=\sum_{n=1}^{\infty} \frac{2 v_{0}^{2} l \tau_{n}^{2}}{1+\gamma_{R} \tau_{n}}\left(1-\frac{e^{-\gamma_{R} t}-\gamma_{R} \tau_{n} e^{-t / \tau_{n}}}{1-\gamma_{R} \tau_{n}}\right) .
$$

For times $\gamma_{R} t \ll 1$ and $t / \tau_{n} \ll 1$, the Taylor expansion of the exponential functions yields

$$
\left\langle\overline{\Delta \boldsymbol{r}_{a}^{2}}(t)\right\rangle=v_{0}^{2} l \gamma_{R} t^{2} \sum_{n=1}^{\infty} \frac{\tau_{n}}{1+\gamma_{R} \tau_{n}} .
$$

Thus, we obtain a quadratic time dependence independent of the polymer stiffness. Considering flexible polymers and large Péclet numbers, the relaxation times are given by Eq. (20) and the evaluation of the sum yields for $p L \gg 1$,

$$
\left\langle\overline{\Delta \boldsymbol{r}_{a}^{2}}(t)\right\rangle=\gamma_{R}^{2} P e^{2} \sqrt{\frac{l^{3}}{96 p \mu \Delta}} t^{2} .
$$

This expression quantitatively agrees with the numerical results of the ballistic regime presented in Fig. 8 .

In the time regime $1<\gamma_{R} t \ll \gamma_{R} \tau_{1}$, the sum in Eq. (33) is dominated by contributions with large $n$ and it can conveniently be replaced by an integral, ${ }^{80}$ which yields

$$
\begin{aligned}
\left\langle\overline{\Delta \boldsymbol{r}_{a}^{2}}(t)\right\rangle= & 2 v_{0}^{2} l \tau_{1}^{2}\left(\frac{t}{\tau_{1}}\right)^{3 / 2} \\
& \times \int_{0}^{\infty} d x \frac{x^{2}\left(1-e^{-\gamma_{R} t}\right)-\gamma_{R} t\left(1-e^{-x^{2}}\right)}{x^{2}\left(x^{4}-\left(\gamma_{R} t\right)^{2}\right)},
\end{aligned}
$$

with the definition $x=\sqrt{t / \tau_{1}} n$. Here, we again insert the relaxation times Eq. (20) of a flexible polymer. Since $\gamma_{R} t>1$ and the integral is dominated by contributions for $x<1$, we obtain

$$
\begin{aligned}
\left\langle\overline{\Delta \boldsymbol{r}_{a}^{2}}(t)\right\rangle & =\frac{2 v_{0}^{2} l}{\gamma_{R}} \sqrt{\tau_{1} t} \int_{0}^{\infty} d x \frac{1-e^{-x^{2}}}{x^{2}} \\
& =\frac{\gamma_{R} l^{3} P e^{2}}{2 L} \sqrt{\pi \tau_{1} t}
\end{aligned}
$$

in agreement with the full numerical results presented in Fig. 8.

Inequality (27) implies a strong dependence of the internal dynamics on the number of active sites. This is illustrated in Fig. 9 for $P e=10^{2}$. At large $L / l \gtrsim 10^{3}$, we find an intermediate time regime close to $\sqrt{t}$. This regime gradually vanishes with decreasing $L / l$ and for $L / l \lesssim 10^{2}$ is replaced by a direct crossover from the ballistic motion to free diffusion. A similar behavior is obtained for other Péclet numbers. This effect is particularly important for simulations of polymers 


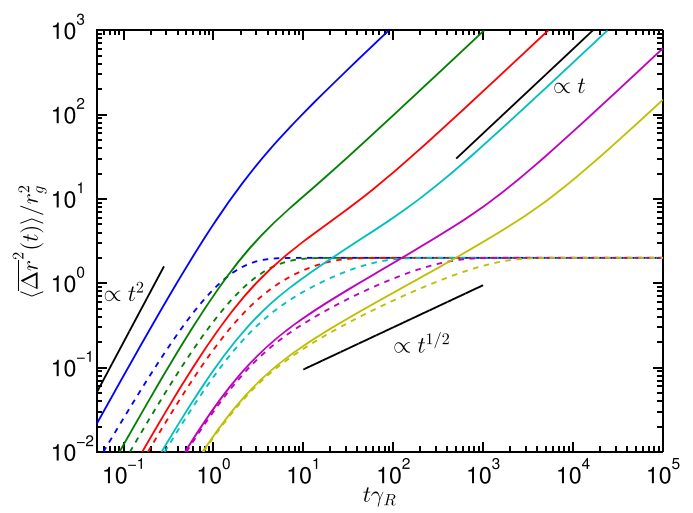

FIG. 9. Mean square displacements of flexible polymers with $P e=10^{2}$ for the numbers of active sides $L / l=2 \times 10^{1}$ (blue), $5 \times 10^{1}$ (green), $10^{2}$ (red), $2 \times 10^{2}$ (cyan), $5 \times 10^{2}$ (purple), and $10^{3}$ (yellow). The time is scaled by the factor $\gamma_{R}=2 D_{R}$ of the rotational diffusivity and the MSD by the radius of gyration $r_{g}$. The dashed lines correspond to the MSDs in the polymer center-of-mass reference frame.

composed of discrete monomers, either ABPs or polymers exposed to APB suspensions. Here, typically, "short" polymers are considered, with $L / l \lesssim 10^{2}{ }^{42,45,48}$ Hence, in such systems, the internal dynamics will only be visible at small $\mathrm{Pe}$.

\section{SUMMARY AND CONCLUSIONS}

We have presented results for the dynamics of active semiflexible polymers, specifically the end-to-end vector relaxation behavior and mean square displacements. We describe the polymer by the Gaussian semiflexible polymer approach, taking its finite extensibility into account in a mean-field manner by a constraint on the total contour length. ${ }^{38-40}$ Activity is modelled as a colored noise force with an exponential temporal correlation. Due to the linearity of the intramolecular forces, the equation of motion can be solved analytically (Ornstein-Uhlenbeck process). ${ }^{86}$

As already discussed in Ref. 10, the finite contour length strongly affects the relaxation times of the polymers. Thereby, the relaxation times decrease substantially with increasing Péclet number for both flexible and semiflexible polymers. This is evident from Fig. 4 for a fixed $p L$ value and Fig. 4 of Ref. 10, where $\tau_{1}$ is shown as a function of the Péclet number for various $p L$ values. In fact, the reduction for semiflexible polymers is stronger than that for flexible ones, and the relaxation times of flexible polymers are assumed for large $P e$ and low mode numbers. The latter is seen in Fig. 2 and even more in Fig. 5 of Ref. 10. This is a major and significant difference to bare Rouse-type models, ${ }^{41-43,58,70}$ where the polymer relaxation times are independent of activity.

For flexible polymers, an increasing activity leads to their monotonic swelling. However, the dominance of the Rousetype modes for semiflexible polymers at large Péclet numbers has pronounced consequences for their conformational properties. Conformational restrictions by bending energy become negligible compared to entropic contributions due to active noise, and the prevalent flexible modes imply shrinkage of semiflexible polymers. ${ }^{10}$ Here, our analytical model provides an explanation for the shrinkage observed in simulations. ${ }^{42,69}$
Moreover, the dominance of the Rouse modes leads to a swelling of semiflexible polymers identical to that of flexible polymers for $\mathrm{Pe} \rightarrow \infty$, and the mean square end-to-end distance saturates at $L^{2} / 2$. This swelling is distinctively different from the stiffening of a passive polymer for $p L \rightarrow 0$. Not only is the fluctuation spectrum different-for a passive semiflexible polymer, bending modes dominate, whereas for the active polymer the flexible modes dominate-but all relaxation times (cf. Eq. (20)) decrease with increasing $P e(\mu \rightarrow \infty$ for $P e \rightarrow \infty$ ), whereas a rod-like polymer exhibits a finite rotation-relaxation time. It is this aspect which implies mean square end-to-end distances smaller than the fully stretched value $L^{2}$.

The correlation function of the polymer end-to-end vector exhibits two decay processes. On the one hand, the polymer relaxation times determine the decay of the correlation for a large number $L / l$ of active sites along the polymer contour independent of polymer stiffness, essentially for all suitable Péclet numbers. On the other hand, for small $L / l$, the decay is determined by the rotational diffusion coefficient of an individual $\mathrm{ABP}$. Polymers with in-between $L / l$ show a crossover from a polymer relaxation-time dominated decay at small Péclet numbers to a decay governed by the rotational diffusion coefficient at large $P e$. In the first case, the decay of the correlation function depends on both the active velocity $v_{0}$ and the rotational diffusion coefficient. In the second case, only the diffusion coefficient is relevant. ${ }^{43,44}$ In simulations of polymers comprised of discrete beads exposed to the colored noise of Eq. (4), e.g., a bath of ABPs, this implies that the relaxation behavior of (very) short polymers is governed by the rotational diffusion coefficient of an individual $\mathrm{ABP}, 43,44$ whereas the relaxation behavior of long polymers is regulated by the internal, activity-dependent relaxation times of the polymer.

As noted earlier, ${ }^{41,42,58}$ the diffusivity of active polymers is substantially enhanced compared to passive polymers, as reflected in mean square displacements. However, the magnitude of the displacements is somewhat smaller in our case compared to calculations neglecting the length constraint. The respective smaller relaxation times yield smaller amplitudes for the various modes (cf. Eq. (31)). Free diffusion of the polymer follows for times $t / \tau_{1} \gg 1$. Since $\tau_{1}$ decreases with increasing Péclet number, the crossover occurs at smaller times for larger activity. This is another major difference to calculations neglecting the length constraint. Since activity gives rise to a ballistic regime in the mean square displacement for $\Gamma_{R} t \lesssim 1$, an increase of the activity via $v_{0}$ leads to a shortening of the time regime dominated by the internal polymer dynamics and a direct crossover can appear between ballistic motion and free diffusion as for an individual ABP. This particularly applies to short polymers.

A major conclusion of our study is that the dynamics of a polymer is determined by the activity for all times as soon as $P e \gg 1$. Aside from the ballistic motion at short times, the dependence of the relaxation time on $P e$ governs the relaxational and translational (MSD) polymer dynamics for $\gamma_{R} t \gtrsim 1$ (cf. Fig. 8). This aspect has not been addressed before, but it is seen in simulations ${ }^{42}$ and experiments. ${ }^{87}$ The exponent characterizing the subdiffusive motion in the MSD is essentially 
independent of activity and persistence length; however, the time range over which subdiffusion persists depends substantially on the activity. We find exponents $\gtrsim 1 / 2$, values larger than those seen in experiments on the dynamics of chromosomal loci. ${ }^{35,36}$ The discrepancy may be related to additional viscoelastic effects of the environment in the experimental studies, as discussed in Ref. 41. However, the propulsion mechanism and its directionality might also matter, which is certainly less isotropic in the experiments and oriented more along the polymer contour. Here, further studies are necessary.

We have neglected hydrodynamic interactions in our considerations. Such interactions modify the dynamical behavior in several ways. On the one hand, we expect a change in the dependence of the relaxation times on the mode number as in the Zimm model. ${ }^{43,80}$ On the other hand, preaveraging might yield an additional dependence on activity, a dependence not accounted for by a "traditional" approach. ${ }^{80}$ Calculations along this line are under way.

\section{ACKNOWLEDGMENTS}

Financial support by the Deutsche Forschungsgemeinschaft (DFG) within the priority program SPP 1726 "Microswimmers-from Single Particle Motion to Collective Behaviour" is gratefully acknowledged.

\section{APPENDIX: APPROXIMATION OF EQ. (17)}

For flexible polymers $(p L \gg 1)$, the sum in Eq. (17) over the mode numbers can be evaluated analytically. With the approximation

$$
\int_{-L / 2}^{L / 2}\left(\frac{d \varphi_{n}(s)}{d s}\right)^{2} d s=\zeta_{n}^{2}
$$

and $\tau_{n}$ of Eq. (16), Eq. (17) becomes

$$
\sum_{n=1}^{\infty} \frac{3}{\epsilon \zeta_{n}^{2}+2 \lambda}+\frac{v_{0}^{2} l \gamma^{2}}{\left(k_{B} T\right)^{2}\left(\epsilon^{2} \zeta_{n}^{6}+4 \lambda \epsilon \zeta_{n}^{4}+4 \lambda^{2} \zeta_{n}^{2}\right)+k_{B} T \gamma \gamma_{R}\left(\epsilon \zeta_{n}^{2}+2 \lambda\right)}=L
$$

Using $\zeta_{n}=n \pi / L$, the sum, evaluated up to order $n^{4}$, yields

$$
\begin{gathered}
\frac{\operatorname{coth}(2 p L \sqrt{\mu})}{\sqrt{\mu}}-\frac{1}{2 p L \mu}+\frac{P e^{2}}{6 \Delta}\left[\sqrt { \frac { 1 } { 2 \mu } } \left(\frac{\sqrt{1-\beta}}{\beta} \operatorname{coth}(p L \sqrt{2 \mu(1-\beta)})\right.\right. \\
\left.\left.-\frac{\sqrt{1+\beta}}{\beta} \operatorname{coth}(p L \sqrt{2 \mu(1+\beta)})\right)+\frac{\operatorname{coth}(2 p L \sqrt{\mu})}{\sqrt{\mu}}-\frac{1}{2 p L \mu}\right]=1
\end{gathered}
$$

with

$$
\beta=\sqrt{1-\frac{2}{3 \Delta l^{3} p^{3} \mu^{2}}}
$$

This expression applies as long as $2 /\left(3 \Delta l^{3} p^{3} \mu^{2}\right)<1$. In case $2 /\left(3 \Delta l^{3} p^{3} \mu^{2}\right)>1$, the introduction of

$$
\tilde{\beta}=i \sqrt{\frac{2}{3 \Delta l^{3} p^{3} \mu^{2}}-1}
$$

and analytical continuation leads to the expression

$$
\begin{aligned}
\frac{1}{\sqrt{\mu}}+ & \frac{P e^{2}}{6 \Delta}\left[\sqrt{\frac{2}{\mu}} \frac{\left(1+\tilde{\beta}^{2}\right)^{1 / 4} \sin (\alpha / 2) \sinh (2 x)}{\tilde{\beta}[\cosh (2 x)-\cos (2 y)]}\right. \\
& \left.-\sqrt{\frac{2}{\mu}} \frac{\left(1+\tilde{\beta}^{2}\right)^{1 / 4} \cos (\alpha / 2) \sin (2 y)}{\tilde{\beta}[\cosh (2 x)-\cos (2 y)]}+\frac{1}{\sqrt{\mu}}\right]=1
\end{aligned}
$$

Here, the abbreviations $\alpha=\arctan (\tilde{\beta}), x=p L \sqrt{2 \mu}\left(1+\tilde{\beta}^{2}\right)^{1 / 4}$ $\cos (\alpha / 2)$, and $y=p L \sqrt{2 \mu}\left(1+\tilde{\beta}^{2}\right)^{1 / 4} \sin (\alpha / 2)$ are used. The limit $p L \gg 1$ implies

$$
1=\frac{1}{\sqrt{\mu}}+\frac{P e^{2}}{6 \Delta}\left[\sqrt{\frac{2}{\mu}} \frac{\left(1+\tilde{\beta}^{2}\right)^{1 / 4}}{\tilde{\beta}} \sin \left(\frac{\alpha}{2}\right)+\frac{1}{\sqrt{\mu}}\right] .
$$

Comparison with the full numerical solution yields excellent agreement.

\footnotetext{
${ }^{1}$ E. Lauga and T. R. Powers, "The hydrodynamics of swimming microorganisms," Rep. Prog. Phys. 72, 096601 (2009).

${ }^{2}$ S. Ramaswamy, "The mechanics and statistics of active matter," Annu. Rev. Condens. Matter Phys. 1, 323 (2010).

${ }^{3}$ T. Vicsek and A. Zafeiris, "Collective motion," Phys. Rep. 517, 71 (2012).

${ }^{4}$ P. Romanczuk, M. Bär, W. Ebeling, B. Lindner, and L. Schimansky-Geier, "Active Brownian particles," Eur. Phys. J.: Spec. Top. 202, 1 (2012).

${ }^{5}$ M. C. Marchetti, J. F. Joanny, S. Ramaswamy, T. B. Liverpool, J. Prost, M. Rao, and R. A. Simha, "Hydrodynamics of soft active matter," Rev. Mod. Phys. 85, 1143 (2013).

${ }^{6}$ J. Elgeti, R. G. Winkler, and G. Gompper, "Physics of microswimmersSingle particle motion and collective behavior: A review," Rep. Prog. Phys. 78, 056601 (2015).

${ }^{7}$ C. Bechinger, R. Di Leonardo, H. Löwen, C. Reichhardt, G. Volpe, and G. Volpe, "Active particles in complex and crowded environments," Rev. Mod. Phys. 88, 045006 (2016).

${ }^{8}$ M. C. Marchetti, Y. Fily, S. Henkes, A. Patch, and D. Yllanes, "Minimal model of active colloids highlights the role of mechanical interactions in
} 
controlling the emergent behavior of active matter," Curr. Opin. Colloid Interface Sci. 21, 34 (2016).

${ }^{9}$ A. Zöttl and H. Stark, "Emergent behavior in active colloids," J. Phys.: Condens. Matter 28, 253001 (2016).

${ }^{10}$ T. Eisenstecken, G. Gompper, and R. G. Winkler, "Conformational properties of active semiflexible polymers," Polymers 8, 304 (2016).

${ }^{11}$ F. J. Nédélec, T. Surrey, A. C. Maggs, and S. Leibler, "Self-organization of microtubules and motors," Nature 389, 305 (1997).

${ }^{12} \mathrm{~J}$. Howard, Mechanics of Motor Proteins and the Cytoskeleton (Sinauer Associates Sunderland, MA, 2001).

${ }^{13}$ K. Kruse, J. F. Joanny, F. Jülicher, J. Prost, and K. Sekimoto, "Asters, vortices, and rotating spirals in active gels of polar filaments," Phys. Rev. Lett. 92, 078101 (2004).

${ }^{14}$ A. R. Bausch and K. Kroy, "A bottom-up approach to cell mechanics," Nat. Phys. 2, 231 (2006).

${ }^{15} \mathrm{~F}$. Jülicher, K. Kruse, J. Prost, and J.-F. Joanny, "Active behavior of the cytoskeleton," Phys. Rep. 449, 3 (2007).

${ }^{16}$ Y. Harada, A. Noguchi, A. Kishino, and T. Yanagida, "Sliding movement of single actin filaments on one-headed myosin filaments," Nature 326, 805 (1987).

${ }^{17}$ V. Schaller, C. Weber, C. Semmrich, E. Frey, and A. R. Bausch, "Polar patterns of driven filaments," Nature 467, 73 (2010).

${ }^{18}$ J. Prost, F. Jülicher, and J.-F. Joanny, "Active gel physics," Nat. Phys. 11, 111 (2015)

${ }^{19}$ H. C. Berg, E. Coli in Motion, Biological and Medical Physics Series (Springer, New York, 2004).

${ }^{20}$ J. R. Howse, R. A. L. Jones, A. J. Ryan, T. Gough, R. Vafabakhsh, and R. Golestanian, "Self-motile colloidal particles: From directed propulsion to random walk," Phys. Rev. Lett. 99, 048102 (2007).

${ }^{21}$ G. Volpe, I. Buttinoni, D. Vogt, H. J. Kümmerer, and C. Bechinger, "Microswimmers in patterned environments," Soft Matter 7, 8810 (2011).

${ }^{22}$ S. Thutupalli, R. Seemann, and S. Herminghaus, "Swarming behavior of simple model squirmers," New J. Phys. 13, 073021 (2011).

${ }^{23}$ I. Buttinoni, J. Bialké, F. Kümmel, H. Löwen, C. Bechinger, and T. Speck, "Dynamical clustering and phase separation in suspensions of self-propelled colloidal particles," Phys. Rev. Lett. 110, 238301 (2013).

${ }^{24}$ B. ten Hagen, F. Kümmel, R. Wittkowski, D. Takagi, H. Löwen, and C. Bechinger, "Gravitaxis of asymmetric self-propelled colloidal particles," Nat. Commun. 5, 4829 (2014).

${ }^{25}$ C. C. Maass, C. Krüger, S. Herminghaus, and C. Bahr, "Swimming droplets," Annu. Rev. Condens. Matter Phys. 7, 171 (2016).

${ }^{26}$ J. Stenhammar, R. Wittkowski, D. Marenduzzo, and M. E. Cates, “Activityinduced phase separation and self-assembly in mixtures of active and passive particles," Phys. Rev. Lett. 114, 018301 (2015).

${ }^{27}$ A. Wysocki, R. G. Winkler, and G. Gompper, "Propagating interfaces in mixtures of active and passive Brownian particles," New J. Phys. 18, 123030 (2016).

${ }^{28}$ L. Angelani, C. Maggi, M. L. Bernardini, A. Rizzo, and R. Di Leonardo, "Effective interactions between colloidal particles suspended in a bath of swimming cells," Phys. Rev. Lett. 107, 138302 (2011).

${ }^{29}$ Y. Peng, L. Lai, Y.-S. Tai, K. Zhang, X. Xu, and X. Cheng, "Diffusion of ellipsoids in bacterial suspensions," Phys. Rev. Lett. 116, 068303 (2016).

${ }^{30}$ K. C. Leptos, J. S. Guasto, J. P. Gollub, A. I. Pesci, and R. E. Goldstein, "Dynamics of enhanced tracer diffusion in suspensions of swimming eukaryotic microorganisms," Phys. Rev. Lett. 103, 198103 (2009).

${ }^{31}$ A. Morozov and D. Marenduzzo, "Enhanced diffusion of tracer particles in dilute bacterial suspensions," Soft Matter 10, 2748 (2014).

${ }^{32}$ C. P. Brangwynne, G. H. Koenderink, F. C. MacKintosh, and D. A. Weitz, "Cytoplasmic diffusion: Molecular motors mix it up," J. Cell Biol. 183, 583 (2008).

${ }^{33}$ C. P. Brangwynne, G. H. Koenderink, F. C. MacKintosh, and D. A. Weitz, "Nonequilibrium microtubule fluctuations in a model cytoskeleton," Phys. Rev. Lett. 100, 118104 (2008).

${ }^{34}$ C. A. Weber, R. Suzuki, V. Schaller, I. S. Aranson, A. R. Bausch, and E. Frey, "Random bursts determine dynamics of active filaments," Proc. Natl. Acad. Sci. U. S. A. 112, 10703 (2015).

${ }^{35}$ S. C. Weber, A. J. Spakowitz, and J. A. Theriot, "Nonthermal ATPdependent fluctuations contribute to the in vivo motion of chromosomal loci," Proc. Natl. Acad. Sci. U. S. A. 109, 7338 (2012).

${ }^{36}$ A. Javer, Z. Long, E. Nugent, M. Grisi, K. Siriwatwetchakul, K. D. Dorfman, P. Cicuta, and M. Cosentino Lagomarsino, "Short-time movement of E. coli chromosomal loci depends on coordinate and subcellular localization," Nat. Commun. 4, 3003 (2013).
${ }^{37}$ A. Zidovska, D. A. Weitz, and T. J. Mitchison, "Micron-scale coherence in interphase chromatin dynamics," Proc. Natl. Acad. Sci. U. S. A. 110, 15555 (2013).

${ }^{38}$ R. G. Winkler, P. Reineker, and L. Harnau, "Models and equilibrium properties of stiff molecular chains," J. Chem. Phys. 101, 8119 (1994).

${ }^{39}$ R. G. Winkler, "Deformation of semiflexible chains," J. Chem. Phys. 118 2919 (2003).

${ }^{40}$ R. G. Winkler, "Semiflexible polymers in shear flow," Phys. Rev. Lett. 97, 128301 (2006)

${ }^{41} \mathrm{H}$. Vandebroek and C. Vanderzande, "Dynamics of a polymer in an active and viscoelastic bath," Phys. Rev. E 92, 060601 (2015).

${ }^{42}$ J. Shin, A. G. Cherstvy, W. K. Kim, and R. Metzler, "Facilitation of polymer looping and giant polymer diffusivity in crowded solutions of active particles," New J. Phys. 17, 113008 (2015).

${ }^{43}$ N. Samanta and R. Chakrabarti, "Chain reconfiguration in active noise," J. Phys. A: Math. Theor. 49, 195601 (2016).

${ }^{44}$ R. G. Winkler, "Dynamics of flexible active Brownian dumbbells in the absence and the presence of shear flow," Soft Matter 12, 3737 (2016).

${ }^{45}$ A. Kaiser, S. Babel, B. ten Hagen, C. von Ferber, and H. Löwen, "How does a flexible chain of active particles swell?," J. Chem. Phys. 142, 124905 (2015).

${ }^{46}$ D. Loi, S. Mossa, and L. F. Cugliandolo, "Non-conservative forces and effective temperatures in active polymers," Soft Matter 7, 10193 (2011).

${ }^{47}$ R. Chelakkot, A. Gopinath, L. Mahadevan, and M. F. Hagan, "Flagellar dynamics of a connected chain of active, polar, Brownian particles," J. R. Soc., Interface 11, 20130884 (2014).

${ }^{48}$ A. Kaiser and H. Löwen, "Unusual swelling of a polymer in a bacterial bath,” J. Chem. Phys. 141, 044903 (2014).

${ }^{49}$ F. Peruani, L. Schimansky-Geier, and M. Bär, "Cluster dynamics and cluster size distributions in systems of self-propelled particles," Eur. Phys. J.: Spec. Top. 191, 173 (2010).

${ }^{50}$ Y. Fily and M. C. Marchetti, "Athermal phase separation of self-propelled particles with no alignment," Phys. Rev. Lett. 108, 235702 (2012).

${ }^{51}$ J. Bialké, T. Speck, and H. Löwen, "Crystallization in a dense suspension of self-propelled particles,” Phys. Rev. Lett. 108, 168301 (2012).

${ }^{52}$ G. S. Redner, M. F. Hagan, and A. Baskaran, "Structure and dynamics of a phase-separating active colloidal fluid," Phys. Rev. Lett. 110, 055701 (2013)

${ }^{53}$ A. Wysocki, R. G. Winkler, and G. Gompper, "Cooperative motion of active Brownian spheres in three-dimensional dense suspensions," EPL 105, 48004 (2014).

${ }^{54}$ R. G. Winkler, A. Wysocki, and G. Gompper, "Virial pressure in systems of spherical active Brownian particles," Soft Matter 11, 6680 (2015).

${ }^{55}$ B. ten Hagen, R. Wittkowski, D. Takagi, F. Kümmel, C. Bechinger, and H. Löwen, "Can the self-propulsion of anisotropic microswimmers be described by using forces and torques?," J. Phys.: Condens. Matter 27, 194110 (2015)

${ }^{56}$ T. B. Liverpool, A. C. Maggs, and A. Ajdari, "Viscoelasticity of solutions of motile polymers," Phys. Rev. Lett. 86, 4171 (2001).

${ }^{57}$ D. Sarkar, S. Thakur, Y.-G. Tao, and R. Kapral, "Ring closure dynamics for a chemically active polymer," Soft Matter 10, 9577 (2014).

${ }^{58}$ A. Ghosh and N. S. Gov, "Dynamics of active semiflexible polymers," Biophys. J. 107, 1065 (2014).

${ }^{59}$ R. E. Isele-Holder, J. Elgeti, and G. Gompper, "Self-propelled worm-like filaments: Spontaneous spiral formation, structure, and dynamics," Soft Matter 11, 7181 (2015).

${ }^{60}$ R. E. Isele-Holder, J. Jager, G. Saggiorato, J. Elgeti, and G. Gompper, "Dynamics of self-propelled filaments pushing a load," Soft Matter 12 8495 (2016)

${ }^{61}$ A. Laskar, R. Singh, S. Ghose, G. Jayaraman, P. B. S. Kumar, and R. Adhikari, "Hydrodynamic instabilities provide a generic route to spontaneous biomimetic oscillations in chemomechanically active filaments," Sci. Rep. 3, 1964 (2013).

${ }^{62}$ G. Jayaraman, S. Ramachandran, S. Ghose, A. Laskar, M. S. Bhamla, P. B. S. Kumar, and R. Adhikari, "Autonomous motility of active filaments due to spontaneous flow-symmetry breaking," Phys. Rev. Lett. 109, 158302 (2012)

${ }^{63} \mathrm{H}$. Jiang and Z. Hou, "Motion transition of active filaments: Rotation without hydrodynamic interactions," Soft Matter 10, 1012 (2014)

${ }^{64} \mathrm{~S}$. Babel, H. Löwen, and A. M. Menzel, "Dynamics of a linear magnetic "microswimmer molecule,"' EPL 113, 58003 (2016).

${ }^{65} \mathrm{C}$. Valeriani, M. Li, J. Novosel, J. Arlt, and D. Marenduzzo, "Colloids in a bacterial bath: Simulations and experiments," Soft Matter 7, 5228 (2011). 
${ }^{66}$ A. Suma, G. Gonnella, D. Marenduzzo, and E. Orlandini, "Motility-induced phase separation in an active dumbbell fluid," EPL 108, 56004 (2014).

${ }^{67}$ L. F. Cugliandolo, G. Gonnella, and A. Suma, "Rotational and translational diffusion in an interacting active dumbbell system," Phys. Rev. E 91, 062124 (2015).

${ }^{68}$ N. Küchler, H. Löwen, and A. M. Menzel, "Getting drowned in a swirl: Deformable bead-spring model microswimmers in external flow fields," Phys. Rev. E 93, 022610 (2016).

${ }^{69}$ J. Harder, C. Valeriani, and A. Cacciuto, "Activity-induced collapse and reexpansion of rigid polymers," Phys. Rev. E 90, 062312 (2014).

${ }^{70}$ D. Osmanovic and Y. Rabin, "Dynamics of active Rouse chains," Soft Matter 13, 963 (2017)

${ }^{71}$ L. Harnau, R. G. Winkler, and P. Reineker, "Dynamic structure factor of semiflexible macromolecules in dilute solution,” J. Chem. Phys. 104, 6355 (1996).

${ }^{72}$ L. Harnau, R. G. Winkler, and P. Reineker, "Influence of stiffness on the dynamics of macromolecules in a melt," J. Chem. Phys. 106, 2469 (1997).

${ }^{73}$ L. Harnau, R. G. Winkler, and P. Reineker, "Dynamic properties of molecular chains with variable stiffness," J. Chem. Phys. 102, 7750 (1995).

${ }^{74}$ R. G. Winkler, L. Harnau, and P. Reineker, "Distribution functions and dynamical properties of stiff macromolecules," Macromol. Theory Simul. 6, 1007 (1997).

${ }^{75}$ R. G. Winkler, "Conformational and rheological properties of semiflexible polymers in shear flow," J. Chem. Phys. 133, 164905 (2010).

${ }^{76}$ R. G. Winkler, "Diffusion and segmental dynamics of rodlike molecules by fluorescence correlation spectroscopy,” J. Chem. Phys. 127, 054904 (2007).
${ }^{77}$ U. M. B. Marconi, M. Paoluzzi, and C. Maggi, "Effective potential method for active particles," Mol. Phys. 114, 2400 (2016).

${ }^{78}$ R. G. Winkler and P. Reineker, "Finite size distribution and partition functions of Gaussian chains: Maximum entropy approach," Macromolecules 25, 6891 (1992)

${ }^{79}$ R. G. Winkler, S. Keller, and J. O. Rädler, "Intramolecular dynamics of linear macromolecules by fluorescence correlation spectroscopy," Phys. Rev. E 73, 041919 (2006).

${ }^{80} \mathrm{M}$. Doi and S. F. Edwards, The Theory of Polymer Dynamics (Clarendon Press, Oxford, 1986).

${ }^{81}$ S. R. Aragón and R. Pecora, "Dynamics of wormlike chains," Macromolecules 18, 1868 (1985).

${ }^{82}$ E. Farge and A. C. Maggs, "Dynamic scattering from semiflexible polymers," Macromolecules 26, 5041 (1993).

${ }^{83}$ E. P. Petrov, T. Ohrt, R. G. Winkler, and P. Schwille, "Diffusion and segmental dynamics of double-stranded DNA," Phys. Rev. Lett. 97, 258101 (2006).

${ }^{84}$ R. Götter, K. Kroy, E. Frey, M. Bärmann, and E. Sackmann, "Dynamic light scattering from semidilute actin solutions: A study of hydrodynamic screening, filament bending stiffness, and the effect of tropomyosin/troponinbinding," Macromolecules 29, 30 (1996).

${ }^{85}$ R. Granek, "From semi-flexible polymers to membranes: Anomalous diffusion and reptation," J. Phys. II 7, 1761 (1997).

${ }^{86}$ H. Risken, The Fokker-Planck Equation (Springer, Berlin, 1989).

${ }^{87}$ D. Humphrey, C. Duggan, D. Saha, D. Smith, and J. Kas, "Active fluidization of polymer networks through molecular motors," Nature 416, 413 (2002). 